\title{
The Latest Study of Gravitational Wave Communication
}

\section{System}

\author{
Yoshinari Minami \\ Advanced Science-Technology Research Organization (Formerly NEC Space Development Division), 35-13, Higashikubo-Cho, \\ Nishi-Ku, Yokohama 220-0062, Japan
}

\begin{abstract}
Gravitational wave is a strain wave of space and this can be also generated by strong magnetic field. The principle of gravitational wave generation using the fluctuation in strain field induced by magnetic field is introduced. Using both foregoing gravitational wave generator and gravitational wave detector (i.e. laser interferometric gravitational wave antenna), the gravitational communication system can be possible. This paper introduces its content presented at 20th Annual Lecture (1989) and the research trends in the latest gravitational wave.
\end{abstract}

Key words: Gravitational wave, space-time, continuum, space strain, strain wave, curvature, gravitational wave communication, magnetic field.

\section{Introduction}

Gravitational wave antennas of the gravitational wave detection have been developed in each country.

However, the detection ability of the faint gravitational waves from a celestial body as the gravitational wave source is not sufficient.

On Feb. 12, 2016, the LIGO (Laser Interferometer Gravitational-Wave Observatory) eventually detected gravitational waves created from the collision between two black holes.

Minami proposed the theoretical method of gravitational wave generation induced by pulse-like strong magnetic field in 1989 [1]. The published paper entitled "A Possibility of Gravitational Wave Communication", which was written in Japanese, was presented at 20th Annual Lecture in "The Japan Society for Aeronautical and Space Sciences" (April 1989).

Gravitational wave is a strain wave of space and this can be also generated by strong magnetic field.

Corresponding author: Yoshinari Minami, administrative director, research fields: satellite design and engineering, propulsion theory and propulsion physics, laser propulsion, interstellar navigation theory. E-mail: y-minami@mtj.biglobe.ne.jp.
Namely, the principle of gravitational wave generation using the fluctuation in strain field induced by strong magnetic field is introduced. Using both foregoing gravitational wave generator and gravitational wave detector (i.e. laser interferometric gravitational wave antenna), the gravitational communication system can be possible.

This paper introduces its content presented at 20th Annual Lecture [1] and the research trends in the latest gravitational wave.

\section{Current Status of Gravitational Wave Antenna}

A gravitational wave is a wave of the gravitational field, its essence is a strain wave of space.

Here, strain is the strain which is defined on the continuum mechanics.

As the electromagnetic wave is generated by the vibration of the charge, the gravitational wave is generated by fluctuations in the gravitational field due to the change in mass distribution.

In other words, when the matter by accelerated motion changes in space, the change of strain propagates through space as a gravitational wave. 
For gravitational wave emission, it is necessary to change the quadrupole moment tensor in time of the mass distribution of the matter. In general terms, gravitational waves are radiated by objects whose motion involves acceleration, provided that the motion is not perfectly spherically symmetric (like an expanding or contracting sphere) or rotationally symmetric (like a spinning disk or sphere). A simple example of this principle is a spinning dumbbell. If the dumbbell spins around its axis of symmetry, it will not radiate gravitational wave; if it tumbles end over end, as in the case of two planets orbiting each other, it will radiate gravitational wave. The heavier the dumbbell, and the faster it tumbles, the greater is the gravitational wave radiation it will give off. In an extreme case, such as when the two weights of the dumbbell are massive stars like neutron stars or black holes, orbiting each other quickly, then significant amounts of gravitational wave radiation would be given off.

However, the generation of gravitational wave is very weak, so the occurrence of the ground is impossible from a strength standpoint. Possible gravitational wave sources, at present, are limited to the celestial phenomenon with an abrupt change.

That is, although they are the burst gravitational wave to be released during the supernova explosion, the star of gravitational collapse and the continuous gravitational wave released by binary pulsar, their strength are even so very weak. The strength of the gravitational wave that reaches the Earth is said to be about $h=10^{-29}$ to $h=10^{-25}$. Here " $h$ " denotes the amplitude of the average gravitational wave in general.

On the other hand, in order to detect gravitational wave, the spacing of the two mass points that varies by the passage of gravitational wave is utilized, as a kind of tidal effect.

For example, they are the resonant antenna type detector for detecting the small vibrations of the gravitational wave antenna that gravitational wave causes, the laser interferometric detector which the change in the distance between the mass point is detected directly by using the light interference of the laser, satellite Doppler tracking detection, and the like; various gravitational wave antennas have been developed. However, the detection sensitivity is about $h=1.1 \times 10^{-15}-2.1 \times 10^{-18}$. Gravitational wave detection sensitivity of the current world's highest sensitivity is $h=2.1 \times 10^{-18}$ (in 1989). In this way, since there is the considerable technical problem in the detection sensitivity of the intensity between gravitational wave antenna and gravitational wave from celestial body that is theoretically expected to release, we have not yet succeeded in the detection of gravitational wave (in 1989).

As is well known, the gravitational wave is a transverse wave, has two independent polarization states, then, is similar to the electromagnetic wave. Significant difference is as follows: electromagnetic wave is easily absorbed or scattered by material, but gravitational wave would pass through the material such as a star without attenuation, that is, almost for interaction with the material is extremely small. Since any matter is almost transparent with respect to the gravitational wave, it would be flow-through.

This transparency of goodness brings us the possibility of the direct communication with the other side of the Earth, direct communication between the back side of the moon and the satellite or the Earth.

However, the problem of artificial gravitational wave generation is its extremely low generation efficiency. The intensity of the gravitational wave that can be generated in the laboratory is about $h \leq 10^{-42}$. Thus, the detectable occurrence of gravitational wave due to a change in the quadrupole moment tensor of the matter is almost impossible, then gravitational wave communication is with the status quo difficult from this point. In the following section, the gravitational wave generation method using magnetic field is introduced. 


\section{Gravitational Wave Generation due to the Fluctuation of Strain Field}

\subsection{Gravitational Wave as Strain Wave of Space}

In the first place, we explain about the strain of space $[2,3]$.

Space is an infinite continuum and its structure is determined by Riemannian geometry. The nature of actual physical space is a four-dimensional Riemann space, that is, three dimensional space $\left(x=x^{1}, y=x^{2}\right.$, $\left.\mathrm{z}=\mathrm{x}^{3}\right)$ and one dimensional time $\left(\mathrm{w}=\mathrm{ct}=\mathrm{x}^{0}\right)$, where $c$ is the velocity of light. These four coordinate axes are denoted as $x^{i}(i=0,1,2,3)$.

The square of the infinitesimal distance "ds" between two infinitely proximate points $x^{i}$ and $x^{i}+d x^{i}$ is given by equation of the form:

$$
d s^{2}=g_{i j} d x^{i} d x^{j}
$$

where $g_{i j}$ is a metric tensor.

The metric tensor $g_{\mathrm{ij}}$ determines all the geometrical properties of space and it is a function of this space coordinate. In Riemann space, the metric tensor $\mathrm{g}_{\mathrm{ij}}$ determines a Riemannian connection coefficient $\Gamma^{i}{ }_{j k}$, and furthermore determines the Riemann curvature tensor $R^{p}{ }_{i j k}$ or $R_{p i j k}$, thus the geometry of space is determined by metric tensor.

An external physical action such as the existence of mass energy or electromagnetic energy yields the structural deformation of space. In the deformed space region, the infinitesimal distance is given by:

$$
d s^{\prime 2}=g_{i j}^{\prime} d x^{i} d x^{j}
$$

where $g_{i j}^{\prime}$ the metric tensor of deformed space region, and we use the convected coordinates $\left(x^{\prime i}=x^{i}\right)$.

As shown in Fig. 1, if the line element between the arbitrary two near points (A and B) in space region $\mathrm{S}$ (before structural deformation) is defined as $d s=g_{i} d x^{i}$, the infinitesimal distance between the two near points is given by Eq. (1): $d s^{2}=g_{i j} d x^{i} d x^{j}$.

Let us assume that a space region $\mathbf{S}$ is structurally deformed by an external physical action and transformed to space region $\mathbf{T}$. In the deformed space region $\mathbf{T}$, the line element between the identical two near point (A' and B') of the identical space region newly changes, differs from the length and direction, and becomes $d s^{\prime}=g_{i}^{\prime} d x^{i}$.

Therefore, the infinitesimal distance between the two near points using the convected coordinate $\left(x^{\prime i}=x^{i}\right)$ is given by:

$$
d s^{\prime 2}=g_{i j}^{\prime} d x^{i} d x^{j}
$$

The $g_{i}^{\prime}$ is the transformed base vector from the original base vector $g^{i}$ and the $g_{i j}^{\prime}$ is the transformed metric tensor from the original metric tensor $g_{i j}$. Since the degree of deformation can be expressed as the change of distance between the two points, we get:

$$
\begin{gathered}
d s^{\prime 2}-d s^{2}=g_{i j}^{\prime} d x^{i} d x^{j}-g_{i j} d x^{i} d x^{j} \\
=\left(g_{i j}^{\prime}-g_{i j}\right) d x^{i} d x^{j}=r_{i j} d x^{i} d x^{j}
\end{gathered}
$$

Hence the degree of geometrical and structural deformation can be expressed by the quantity denoted change of metric tensor, i.e.

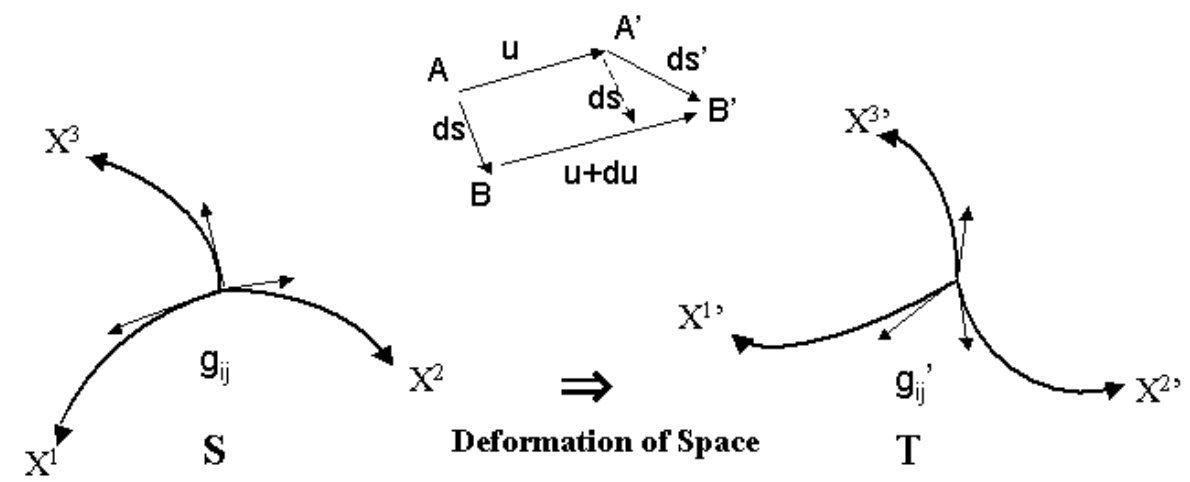

Fig. 1 Fundamental structure of Space. 


$$
r_{i j}=g_{i j}^{\prime}-g_{i j}
$$

On the other hand, the state of deformation can be also expressed by the displacement vector " $u$ " (see Fig. 1).

From the continuum mechanics [4-7], we have the following equations:

$$
\begin{gathered}
d u=g^{i} u_{i: j} d x^{j} \\
d s^{\prime}=d s+d u=d s+g^{i} u_{i: j} d x^{j}
\end{gathered}
$$

Here we use the usual notation ":" for covariant differentiation. As is well known, the partial derivative $u_{i, j}=\frac{\partial u_{i}}{\partial x^{j}}$ is not tensor equation. The covariant derivative $u_{i: j}=u_{i, j}-u_{k} \Gamma_{i j}^{k}$ is tensor equation and can be carried over into all coordinate systems.

From the usual continuum mechanics, the infinitesimal distance after deformation becomes [4]:

$$
\begin{gathered}
d s^{\prime 2}-d s^{2}=r_{i j} d x^{i} d x^{j}= \\
\left(u_{i: j}+u_{j: i}+u_{: i}^{k} u_{k: j}\right) d x^{i} d x^{j}
\end{gathered}
$$

The terms of higher order than second $u^{k}{ }_{: i} u_{k: j}$ can be neglected if the displacement is of small enough value. As the actual physical space can be dealt with the minute displacement from the trial calculation of strain, we get:

$$
r_{i j}=u_{i: j}+u_{j: i}
$$

Whereas, according to the continuum mechanics [4], the strain tensor $e_{i j}$ is given by:

$$
e_{i j}=\frac{1}{2} \cdot r_{i j}=\frac{1}{2} \cdot\left(u_{i: j}+u_{j: i}\right)
$$

So, we get:

$$
d s^{\prime 2}-d s^{2}=\left(g_{i j}^{\prime}-g_{i j}\right) d x^{i} d x^{j}=2 e_{i j} d x^{i} d x^{j}
$$

where $g_{i j}^{\prime}, g_{i j}$ is a metric tensor, $e_{i j}$ is a strain tensor, and $d s^{\prime 2}-d s^{2}$ is a difference of the square of the infinitesimal distance between two infinitely proximate points $x^{i}$ and $x^{i}+d x^{i}$.

Eq. (11) indicates that a certain geometrical structural deformation of space is shown by the concept of strain. In essence, the change of metric tensor $\left(g_{i j}^{\prime}-g_{i j}\right)$ due to the existence of mass energy or electromagnetic energy tensor produces the strain field $e_{i j}$.

Since space-time is distorted, the infinitesimal distance between two infinitely proximate points $x^{i}$ and $x^{i}+d x^{i}$ is important in our understanding of the geometry of the space-time; the physical strain is generated by the difference of a geometrical metric of space-time. Namely, a certain structural deformation is described by strain tensor $e_{i j}$. From Eq. (11), the strain of space is described as follows:

$$
e_{i j}=1 / 2 \cdot\left(g_{i j}{ }^{\prime}-g_{i j}\right)
$$

Now, the metric of flat space where substances are not present is Minkowski metric $\eta_{i j}$. In the case of small curvature limit, that is, supposing that the deviation from the flat space is small enough, the metric of space $g_{i j}$ is given by

$$
g_{i j}=\eta_{i j}+h_{i j}
$$

where $h_{i j}$ is the deviation from the flat space.

$$
\text { From Eqs. (12) and }
$$
$g_{i j}-\eta_{i j}=h_{i j}=\left(g_{i j}{ }^{\prime}-g_{i j}\right)=2 e_{i j}$, then we obtain

$$
e_{i j}=\frac{1}{2} \cdot h_{i j}
$$

As is well known in General Relativity [8-14], using gravitational field equation, Eq. (15) is obtained from the linear equations to the primary of small amount of $h_{i j}$ :

$$
\frac{1}{2} \cdot \square h_{i j}=\square e_{i j}=-\frac{8 \pi G}{c^{4}} \cdot T_{i j}
$$

Namely, the wave equation is obtained by the usual 
method in General Relativity:

$$
\square e_{i j}=\left(\nabla^{2}-\frac{1}{c^{2}} \cdot \frac{\partial^{2}}{\partial t^{2}}\right) \cdot e_{i j}=-\frac{8 \pi G}{c^{4}} \cdot T_{i j}
$$

where $\square$ is the d'Alembert operator (represented by a box: $\square), \mathrm{G}$ is the gravitational constant, c is the light speed and $T_{i j}$ is the energy momentum tensor.

Eq. (16) has a wave solution to propagate at the speed of light, strain of the space $e_{i j}$ in which this propagation is a gravitational wave.

In flat space with no matter, since the energy momentum tensor $T_{i j}$ is zero, the wave equation becomes

$$
\frac{1}{2} \cdot \square h_{i j}=\square e_{i j}=0
$$

Also, the metric tensor in flat space is Minkowski metric at any location, i.e. $\eta_{i j}{ }^{\prime}=\eta_{i j}$, then from Eqs. (10) and (11),

$$
\begin{gathered}
d s^{\prime 2}-d s^{2}=\left(\eta_{i j}{ }^{\prime}-\eta_{i j}\right) d x^{i} d x^{j}= \\
2 e_{i j} d x^{i} d x^{j}=\left(u_{i: j}+u_{j: i}\right) d x^{i} d x^{j}=0
\end{gathered}
$$

Then we get

$$
e_{i j}=0
$$

Further, last term $u_{i: j}+u_{j: i}=0$ indicates Killing equation.

From Eq. (18) or Eq. (19), setting i=j, we get

$$
e_{i i}=u_{i: i}=\operatorname{div} u=0
$$

Since Eq. (20) indicates that the divergence of displacement vector "u", i.e., volume strain is zero: $e_{11}+e_{22}+e_{33}=0$. There exists the following wave equation of space-time satisfying $\operatorname{divu}=0$, that is,

$\nabla^{2} u_{i}-\frac{1}{c^{2}} \cdot \ddot{u}_{i}=0$. And also, this wave motion requires the transversal wave.

The strain wave of space is essentially a transverse wave.

3.2 Generation of Gravitational Wave by Magnetic Field

3.2.1 Spatial Curvature Control by Magnetic Field
Let us consider the electromagnetic energy tensor $M^{i j}$. We apply the electromagnetic energy tensor $M^{i j}$ to gravitational field equation. In this case, the solution of metric tensor $g_{i j}$ is found by

$$
R^{i j}-\frac{1}{2} \cdot g^{i j} R=-\frac{8 \pi G}{c^{4}} \cdot M^{i j}
$$

Eq. (21) determines the structure of space due to the electromagnetic energy.

Here, if we multiply both sides of Eq. (21) by $g_{i j}$, we obtain

$$
\begin{gathered}
g_{i j}\left(R^{i j}-\frac{1}{2} \cdot g^{i j} R\right)= \\
g_{i j} R^{i j}-\frac{1}{2} \cdot g_{i j} g^{i j} R=R-\frac{1}{2} \cdot 4 R=-R \\
g_{i j}\left(\frac{-8 \pi G}{c^{4}} \cdot M^{i j}\right)=-\frac{8 \pi G}{c^{4}} \cdot g_{i j} M^{i j}= \\
\frac{-8 \pi G}{c^{4}} \cdot M_{i}^{i}=\frac{-8 \pi G}{c^{4}} M
\end{gathered}
$$

The following equation is derived from Eqs. (22) and (23):

$$
R=\frac{8 \pi G}{c^{4}} \cdot M
$$

Substituting Eq. (24) into Eq. (21), we obtain

$$
\begin{gathered}
R^{i j}=-\frac{8 \pi G}{c^{4}} \cdot M^{i j}+\frac{1}{2} \cdot g^{i j} R= \\
-\frac{8 \pi G}{c^{4}} \cdot\left(M^{i j}-\frac{1}{2} \cdot g^{i j} M\right)
\end{gathered}
$$

Using antisymmetric tensor $f_{i j}$ which denotes the magnitude of electromagnetic field, the electromagnetic energy tensor $M^{i j}$ is represented as follows:

$$
\begin{gathered}
M^{i j}=-\frac{1}{\mu_{0}} \cdot\left(f^{i \rho} f_{\rho}^{j}-\frac{1}{4} \cdot g^{i j} f^{\alpha \beta} f_{\alpha \beta}\right), \\
f^{i \rho}=g^{i \alpha} g^{\rho \beta} f_{\alpha \beta}
\end{gathered}
$$

Therefore, for M, we have 


$$
\begin{gathered}
M=M_{i}^{i}=g_{i j} M^{i j}= \\
-\frac{1}{\mu_{0}} \cdot\left(g_{i j} f^{i \rho} f_{\rho}^{j}-\frac{1}{4} \cdot g_{i j} g^{i j} f^{\alpha \beta} f_{\alpha \beta}\right) \\
=-\frac{1}{\mu_{0}} \cdot\left(f^{i \rho} f_{i \rho}-\frac{1}{4} \cdot 4 f^{\alpha \beta} f_{\alpha \beta}\right)= \\
-\frac{1}{\mu_{0}} \cdot\left(f^{i \rho} f_{i \rho}-f^{i \rho} f_{i \rho}\right)=0
\end{gathered}
$$

Accordingly, substituting $M=0$ into Eq. (25), we get

$$
R^{i j}=-\frac{8 \pi G}{C^{4}} \cdot M^{i j}
$$

Although Ricci tensor $R^{i j}$ has 10 independent components, the major component is the case of $i=j=0$, i.e., $R^{00}$. Therefore, Eq. (28) becomes

$$
R^{00}=-\frac{8 \pi G}{c^{4}} \cdot M^{00}
$$

On the other hand, 6 components of antisymmetric tensor $f_{i j}=-f_{j i}$ are given by electric field $\mathrm{E}$ and magnetic field B from the relation to Maxwell's field equations:

$$
\begin{gathered}
f_{10}=-f_{01}=\frac{1}{c} \cdot E_{x}, f_{20}=-f_{02}=\frac{1}{c} \cdot E_{y} \\
f_{30}=-f_{03}=\frac{1}{c} E_{z}, f_{12}=-f_{21}=B_{z} \\
f_{23}=-f_{32}=B_{x}, f_{31}=-f_{13}=B_{y} \\
f_{00}=f_{11}=f_{22}=f_{33}=0
\end{gathered}
$$

Substituting Eq. (30) into Eq. (26), we get

$$
M^{00}=-\left(\frac{1}{2} \cdot \varepsilon_{0} E^{2}+\frac{1}{2 \mu_{0}} \cdot B^{2}\right) \approx-\frac{1}{2 \mu_{0}} \cdot B^{2} .
$$

Finally, from Eqs. (29) and (31), we have

$$
R^{00}=\frac{4 \pi G}{\mu_{0} c^{4}} \cdot B^{2}=8.2 \times 10^{-38} \cdot B^{2} \quad(\text { BinTesla })
$$

where $\quad \mu_{0}=4 \pi \times 10^{-7}(H / m)$, $\varepsilon_{0}=1 /(36 \pi) \times 10^{-9}(\mathrm{~F} / \mathrm{m}) \quad, \quad c=3 \times 10^{8}(\mathrm{~m} / \mathrm{s})$, $G=6.672 \times 10^{-11}\left(N \cdot \mathrm{m}^{2} / \mathrm{kg}^{2}\right), \quad B \quad$ is a magnetic field in Tesla and $R^{00}$ is a major component of spatial curvature $\left(1 / \mathrm{m}^{2}\right)$.

Eq. (32) indicates that the major component of spatial curvature can be controlled by magnetic field.

The relationship between curvature and magnetic field was derived by Minami and introduced it in 16th International Symposium on Space Technology and Science (1988) [15].

Eq. (32) is derived from general method.

On the other hand, Levi-Civita also investigated the gravitational field produced by a homogeneous electric or magnetic field, which was expressed by Pauli [16] (see Appendix A).

Here for convenience, we rewrite Eq. (28) or Eq. (32) to co-variant form:

$$
\begin{gathered}
R_{i j}=-\frac{8 \pi G}{c^{4}} \cdot M_{i j} \\
R_{00}=\frac{4 \pi G}{\mu_{0} c^{4}} \cdot B^{2}=8.2 \times 10^{-38} \cdot B^{2} \quad(\text { BinTesla })
\end{gathered}
$$

In contrast above, the major component of spatial curvature by material energy tensor is given by:

$$
R_{00}=\frac{8 \pi G}{c^{2}} \cdot \rho
$$

Since the major component of spatial curvature $R_{00}$ is independent of the cause of material energy and electromagnetic energy as a source, the following equation is obtained:

$$
R_{00}=\frac{4 \pi G}{\mu_{0} c^{4}} \cdot B^{2}=\frac{8 \pi G}{c^{2}} \cdot \rho
$$

This yields

$$
\frac{B^{2}}{2 \mu_{0}}=\rho c^{2}=w
$$

Since $B^{2} / 2 \mu_{0}$ shows the energy density of 
magnetic field $\mathrm{B}\left(\mathrm{J} / \mathrm{m}^{3}\right)$, there is a following relationship to the rest mass density $\rho\left(\mathrm{kg} / \mathrm{m}^{3}\right)$,

$$
w=\rho c^{2}
$$

Eq. (37) is intended to guarantee the relationship E $=\mathrm{mc}^{2}$ of mass and energy.

3.2.2 Generation of Spatial Strain Wave by Magnetic Field

From electromagnetic energy tensor $M_{i j}$ and curvature tensor $R_{i j}$ (Ricci tensor), we obtain:

$$
\begin{gathered}
\frac{1}{2} \cdot \square h_{i j}=-\frac{8 \pi G}{c^{4}} \cdot M_{i j} \\
R_{i j}=-\frac{8 \pi G}{c^{4}} \cdot M_{i j} \\
\frac{1}{2} \cdot \square h_{i j}=R_{i j}\left(\square e_{i j}=R_{i j}\right)
\end{gathered}
$$

In the wave equation Eq. (40), the time variation of $h_{i j}$ depends on the temporal variation of curvature $R_{i j}$ of space. That is, since the curvature of space is varied in terms of time, the strain field of space $e_{i j}=\frac{1}{2} \cdot h_{i j}$ is temporally change, then the strain wave of space due to the fluctuation in strain field is generated.

And, the variation of the major curvature of space corresponds to the variation of the material energy tensor or electromagnetic energy tensor from Eq. (35).

Concerning the solution of the wave equation Eq. (40) for any of $R_{i j}$, the wave solution of outward is determined by the delay potential as with the electromagnetic wave:

$$
h_{i j}=\frac{4 G}{c^{4} r} \int R_{i j}(c t-r, x) d V
$$

As is well known in gravitational wave, in the wave equation in the empty space-time with no matter, the deviation from the flat space $h_{i j}$ has 10 independent components.
Then let's think about gravitational wave radiation. In the case of $i=j=0$, Eq. (40) becomes

$$
\frac{1}{2} \cdot \square h_{00}=R_{00}
$$

that is, $\frac{1}{2} \cdot\left(\nabla^{2} h_{00}-\frac{1}{c^{2}} \cdot \frac{\partial^{2} h_{00}}{\partial t^{2}}\right)=R_{00}$

On the other hand, we have

$$
R_{00}=\frac{4 \pi G}{\mu_{0} c^{4}} \cdot B^{2}=8.2 \times 10^{-38} \cdot B^{2}
$$

Then, applying ON/OFF pulses of magnetic field B or sine wave oscillating current like Eq. (45), we can get time change of major component of spatial curvature $R_{00}$ :

$$
B_{m}=I_{m} e^{i(k x-\omega t)}
$$

where $I_{m}$ is current, $k$ is wave number, $\omega$ is angular frequency.

Namely, the variation in strain field of space is conclusively obtained by variation of the magnetic field.

Further, it is possible to generate a gravitational wave directly by varying equivalent magnetic field just as the distribution control of quadrupole moment tensor of the object.

Even if the gravitational wave does not generate by magnetic field as wave, fluctuation in gravitational field can be generated by magnetic field in the vicinity.

\subsection{Gravitational Wave Radiation Method by Magnetic Field}

The strain wave of space induced by the variation of magnetic field is generated as a wave and propagates as a so-called gravitational wave.

However, gravitational wave does not occur in case that the object body varies to spherically symmetric, because there is no time variation of the quadrupole moment tensor. In this regard, the variation in 
gravitational field can be obtained, but the gravitational wave as wave is not generated. In order to generate gravitational wave, it is necessary to vary strained asymmetrically.

Asymmetric distribution configuration or unbalanced magnetic field current control of the magnetic field is necessary like that the mass of object so as to vary asymmetrically. So, the control of magnetic field such as the variation of quadrupole moment tensor is required. In this case, gravitational wave that propagates as a wave is obtained.

By the way, the gravitational wave radiation due to the current control and the like will have a feature of gravitational wave of very high frequency compared to the frequency of the gravitational wave generated by celestial body.

Although the numerical order of gravitational wave generated by magnetic field is under consideration, if a possible oscillator of gravitational wave is developed in the laboratory, by applying modulation techniques to the weak gravitational wave from celestial bodies, it is expected to increase the gravitational wave detection sensitivity.

\section{Gravitational Wave Communication}

In the case of gravitational wave generated by the object mass, the quadrupole moment tensor $\mathrm{A}_{\mathrm{ij}}$ and the gravitational wave emission power $\mathrm{P}$ are given by the following equation:

$$
\begin{gathered}
A_{i j}=\int \rho\left(x_{i} x_{j}-1 / 3 \cdot \delta_{i j} r^{2}\right) d x d y d z \\
P=\frac{G}{5 c^{2}} \cdot\left(\dddot{A}_{i j}\right)^{2}=\frac{G}{5 c^{2}}\left(\frac{d^{3} A_{i j}}{d t^{3}}\right)^{2}
\end{gathered}
$$

If the cylindrical object is rotated about a vertical axis passing through its center of gravity, the gravitational wave emission power $\mathrm{P}(\mathrm{W})$ is given by the following equation:

$$
P=\frac{32 G I^{2} \omega^{6}}{5 c^{5}}=1.73 \times 10^{-52} I^{2} \omega^{6}
$$

where $\mathrm{I}$ is the inertia moment of cylindrical object, $\omega$ is the angular velocity, $G=6.672 \times 10^{-11}\left(\mathrm{~N} \cdot \mathrm{m}^{2} / \mathrm{kg}^{2}\right)$, c is the light speed.

Given the binary stars of mass $M$ draw a circular orbit around a common center of gravity, the gravitational wave emission power $\mathrm{P}(\mathrm{W})$ is given by:

$$
P=\frac{8 G}{5 c^{5}} M^{2} a^{4} \omega^{6}
$$

where "a" is the distance between two stars and $\omega$ is the angular velocity.

Now, the strength of gravitational wave is indicated by the deviation of the metric tensor $h_{i j}$ from the metric of the flat space (Minkowski metric $\eta_{i j}$ ). That is, $g_{i j}=\eta_{i j}+h_{i j}$. In the case of $\mathrm{i}=\mathrm{j}=0$,

$$
g_{00}=\eta_{00}+h_{00}=-1+h_{00}=-1-\frac{2}{c^{2}} \Phi
$$

then

$$
h_{00}=-\frac{2 \Phi}{c^{2}}
$$

By the presence of a gravitational potential $\Phi, \mathrm{g}_{00}$ deviates from its flat value of -1 .

Meanwhile, the wave solution truly a physical reality by the coordinate transformation is only two spatial components of $h_{11}=-h_{22}$ and $h_{12}=h_{21}$, as is well explained in General Relativity [8-11].

The intensity of the gravitational wave is given by the following equation expressed by $h$ as the amplitude of the average gravitational wave:

$$
h=\sqrt{\left|h_{11}\right|^{2}+\left|h_{12}\right|^{2}}
$$

On the other hand, the energy flux of gravitational wave $S$ (the flow of energy per unit time across a unit area perpendicular to the flow) becomes:

$$
S=\frac{c^{5} k^{2}}{32 \pi G} \cdot\left(\left|h_{11}\right|^{2}+\left|h_{12}\right|^{2}\right)\left(\mathrm{J} / \mathrm{m}^{2} \mathrm{~s}=\mathrm{W} / \mathrm{m}^{2}\right)
$$

where $k=\omega / c=2 \pi f / c$. 
From Eqs. (51) and (52), $\mathrm{S}$ is given by:

$$
S=\frac{c^{5} k^{2}}{32 \pi G} \cdot h^{2}=\frac{c^{3} \pi f^{2}}{8 G} \cdot h^{2}
$$

Meanwhile, the energy flux of gravitational wave $S$ is obtained by the following equation to be received from the gravitational wave source at a distance " $r$ " away from the Earth (with Eq. (53) and Eq. (49)):

$$
S=\frac{P}{4 \pi r^{2}}=\frac{c^{3} \pi f^{2}}{8 G} \cdot h^{2}
$$

Then we get the $h$ as the amplitude of the average gravitational wave as follows:

$$
h=\frac{\sqrt{2 G / c^{3}}}{\pi f} \cdot \frac{\sqrt{P}}{r}
$$

From Eq. (36), we have the relation between magnetic field $\mathrm{B}$ and mass density $\rho$ :

$$
B=\sqrt{2 \mu_{0}} \cdot c \sqrt{\rho}
$$

So, although it may be indirect method, we can predict the intensity of required magnetic field $\mathrm{B}$ to detect gravitational wave using the gravitational wave antenna.

As has been well described in the book of General Relativity [8-11], for example, when the binary star of the same mass as the sun $\left(\mathrm{M}=2 \times 10^{30} \mathrm{~kg}\right)$ at a distance 100 parsecs from the Earth is around the circular orbit (period 0.4 seconds) with a diameter of $1,000 \mathrm{~km}$, the gravitational wave emission power $\mathrm{P}$ (W) is $2.6 \times 10^{39} \mathrm{~W}$ from Eq. (49), and using Eq. (54) the intensity of the gravitational wave at the Earth is obtained $h \approx 10^{-18}$.

If the mass density is known from the mass of binary stars, we can calculate the magnetic field $\mathrm{B}$, which corresponds to $h \approx 10^{-18}$. So we may be able to generate this gravitational wave or change of the gravitational field induced by magnetic field and to detect them by the gravitational wave antenna. The gravitational wave oscillation experiment can be expected.
Since the near-field close to a gravitational wave source does not have a property as a wave, in order to capture as radiation, it is necessary to measure at least one wave length of about away from the gravitational wave source.

If the cylindrical object is rotated about a vertical axis passing through its center of gravity, the gravitational wave emission power $\mathrm{P}(\mathrm{W})$ is calculated from Eq. (48). Here setting mass of 200 tons $\left(2 \times 10^{5}\right.$ $\mathrm{kg}$ ) at angular speed of $\omega=50 \mathrm{rad} \mathrm{s}^{-1}$ and the cylindrical object of $10 \mathrm{~m}$ length, the inertia moment of cylindrical object I is given by:

$$
I=\int \rho x^{2} d V=\left(\frac{M L^{2}}{12}\right)^{2}
$$

From Eqs. (48) and (57), gravitational wave emission power $\mathrm{P}(\mathrm{W})$ becomes $7.8 \times 10^{-30} \mathrm{~W}$.

In the above example, the frequency of occurrence gravitational wave is about $8 \mathrm{~Hz}$, it is necessary to measure at a distance $\lambda=3.8 \times 10^{7} \mathrm{~m}$. The energy of the gravitational wave passing per unit area of the receiving side is $\mathrm{S}=4.3 \times 10^{-48}\left(\mathrm{~W} / \mathrm{m}^{2}\right)$. Then, the amplitude of the gravitational wave becomes $h=6.5 \times 10^{-43}$ from Eq. (55).

Since $h=6.5 \times 10^{-43}$ is too small, then it is not detectable in the gravitational wave detector of the highest sensitivity of the current situation. In this way, in the experimental level, because the gravitational wave generated by the object mass does not extend to a detectable level, gravitational wave communication is said to be difficult.

However, since the curvature of space can be also generated by magnetic field, pseudo-gravitational wave as the variation in strain field or gravitational wave as strain wave of space can be generated. The amplitude $h$ of gravitational wave can be controlled by magnetic field, and also a fairly high frequency of gravitational wave can be possible.

The exact order is currently under consideration, but the amplitude of the gravitational wave $h \approx 10^{-24}$ is expected to be generated by the magnetic field of 
20,000 Tesla.

At the present stage of technology, the generation of high magnetic fields has been realized as follows:

(1) 20-60 Tesla: superconductivity;

(2) 360 Tesla: magnetic flux-compression by electromagnetic force;

The electromagnetic flux-compression is a method to compress magnetic flux by an imploding metal ring (linear) which is accelerated by electromagnetic force provided by a large pulsed current in a primary coil outside. A seed magnetic field to be compressed is injected in advance by a pair of solenoid coils.

(3) 400 Tesla: one-turn coil connected to strong laser produced plasma;

(4) 1,400-2,500 Tesla: magnetic flux compression by chemical explosion or laser ablation method;

(5) $>1$ 1G Tesla: neutron star.

Development of new strong magnetic field generation technology is desired.

As described above, by the combination of the gravitational wave generating device using a magnetic field and the laser interferometer gravitational wave detection device, the verification experiment of gravitational wave communication system is considered to be possible in the near future.

Gravitational wave interaction is extremely small with a matter, so would pass through star without attenuation. So, it is possible to direct satellite communications between the Earth and the other side of the moon.

If the oscillator of gravitational wave is developed, it is expected to increase the gravitational wave detection sensitivity by the application of modulation techniques to the weak gravitational wave from celestial bodies, therefore the development is expected for the future of gravitational wave astronomy.

\section{Trends of Latest Gravitational Wave Detection}

On February 12, 2016, it was published the analysis of advanced LIGO event GW150914 and it was claimed that it was the first direct observation for gravitational waves and black holes at the same time. The signal was named GW150914.

In general, gravitational wave amplitude to be expected is said to be very small, i.e., $h \approx 10^{-29}-10^{-25}$.

On the other hand, during LIGO's fifth Science Run in November 2005, sensitivity reached the primary design specification of a detectable strain of one part in $h=10^{-21}$ over a $100 \mathrm{~Hz}$ bandwidth. LIGO has already attained the detection sensitivity of $h=10^{-22}$. Currently, it is the world's highest sensitivity. Also, the detection sensitivity of Advanced LIGO is said to be $h=10^{-23}$.

Fig. 2 shows the sensitivity for LIGO and LISA (Laser Interferometer Space Antenna). LISA Pathfinder, formerly Small Missions for Advanced Research in Technology-2 (SMART-2), is an ESA spacecraft that was launched on December 3, 2015. The mission will test technologies needed for the eLISA (Evolved Laser Interferometer Space Antenna).

Also in Japan, there exist TAMA 300 and KAGRA (Kamioka Gravitational Wave Detector). TAMA 300 was a gravitational wave detector located at the Mitaka campus of the National Astronomical Observatory of Japan, and is currently working on. The KAGRA is a project of the gravitational wave and is currently developed. The target detection sensitivity of KAGRA is $h \approx 10^{-23}-h \approx 10^{-24}$.

As shown in Fig. 2, the recent detection sensitivity of gravitational wave progresses as compared to previously mentioned. That is, the amplitude of the gravitational wave $h=10^{-22}$ comes possible at present, compared to $h=2.1 \times 10^{-18}$ in 1989 . In this way, the detection sensitivity is increased four-digit.

Since the current sensitivity of gravitational wave antenna has already reached $h=10^{-22}$, the amplitude of the gravitational wave $h \approx 10^{-22}$ is expected to be generated by the magnetic field of 200,000 Tesla, further $h \approx 10^{-24}$ is expected to be generated by the magnetic field of 20,000 Tesla (exact order is 


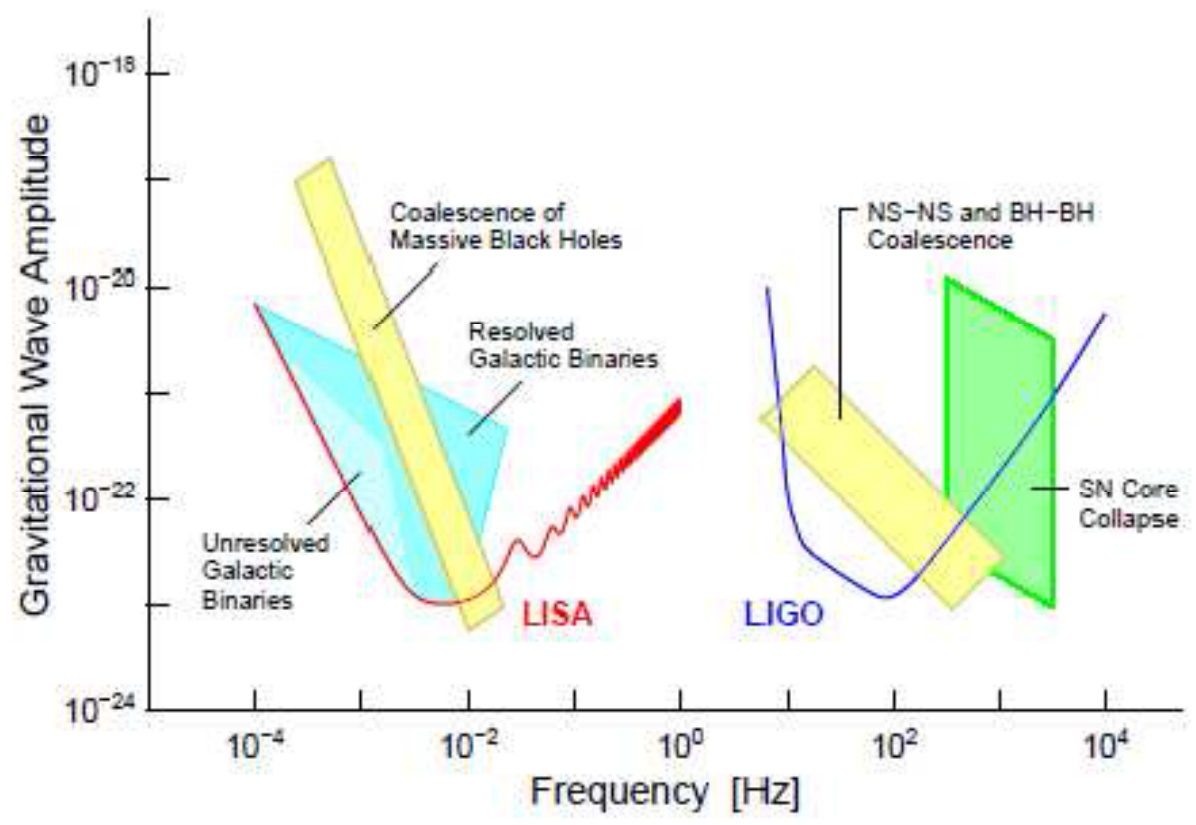

Fig. 2 Sensitivity for LIGO and LISA (Atsushi Nishizawa: Kyoto University; 0804-gasshuku).

currently under consideration). Namely, if KAGRA will attain $h \approx 10^{-24}$ or less of the detection sensitivity in the near future, the gravitational wave generated by the magnetic field of 20,000 Tesla may be directly detected.

\section{Conclusions}

The amplitudes of gravitational wave are ultimately small and the gravitational wave passing through the Earth is many sextillion times weaker than $h \approx 10^{-20}$. Thus, it is difficult that interferometric gravitational wave detectors observe them.

Although gravitational wave is radiated by objects whose motion involves acceleration, its amplitudes of gravitational wave are ultimately small. Accordingly, gravitational wave communication seems to be impossible.

However, the generation of gravitational wave can be possible by magnetic field. Further, recently, some detectors are in the upgrades toward the detection. So, the combination of the gravitational wave generating device using a magnetic field and the laser interferometer gravitational wave detection device may yield the possibility of verification of gravitational wave communication system in the near future at the experimental level.

\section{References}

[1] Minami, Y. 1989. "A Possibility of Gravitational Wave Communication." 20th Annual Lecture in the Japan Society for Aeronautical and Space Sciences.

[2] Minami, Y. 2015. "Continuum Mechanics of Space Seen from the Aspect of General Relativity-An Interpretation of the Gravity Mechanism." Journal of Earth Science and Engineering 5: 188-202.

[3] Williams, C., (Ed.) Minami, Y., (Chap. 3) et al. 2015. Advances in General Relativity Research. Nova Science Publishers.

[4] Flügge, W. 1972. Tensor Analysis and Continuum Mechanics. New York: Springer-Verlag Berlin Heidelberg.

[5] Fung, Y. C. 2001. Classical and Computational Solid Mechanics. World Scientific Publishing Co. Pre. Ltd.

[6] Hans, Z. 1977. An Introduction to Thermomechanics. North-Holland Publishing Company.

[7] Borg, S. F. 1963. Matrix-Tensor Methods in Continuum Mechanics. D. Van Nostrand Company.

[8] Hirakawa, H. 1971. Relativity. Tokyo: Kyoritsu Shuppan Co., Ltd.

[9] Foster, J., and Nightingale, J. D. 1979. General Relativity. Longman Group Ltd.

[10] Tsubono, K. 1986. Ripples of Space-Time. Maruzen Publishing Co., Ltd. 
[11] Misner, C. W., Thorne, K. S., and Wheeler, J. A. 1973. Gravitation. New York: W. H. Freeman and Company.

[12] Tolman, R. C. 1987. Relativity Thermodynamics and Cosmology. New York: Dover Books.

[13] Ryden, B. 2003. Introduction to Cosmology. San Francisco, USA: Addison Weslay.

[14] Matsubara, T. 2010. Introduction to Modern Cosmology:
Coevolution of Spacetime and Matter. Tokyo: University of Tokyo Press.

[15] Minami, Y. 1988. "Space Strain Propulsion System." 16th International Symposium on Space Technology and Science (16th ISTS) 1: 125-36.

[16] Pauli, W. 1981. Theory of Relativity. New York: Dover Publications, Inc. 


\section{Appendix A: Curvature Control by Magnetic Field}

As previously described, we have

$$
R^{00}=\frac{4 \pi G}{\mu_{0} c^{4}} \cdot B^{2}=8.2 \times 10^{-38} \cdot B^{2} \quad(B \text { in Tesla })
$$

where we let $\mu_{0}=4 \pi \times 10^{-7}(\mathrm{H} / \mathrm{m}), \varepsilon_{0}=1 /(36 \pi) \times 10^{-9}(\mathrm{~F} / \mathrm{m}), \quad c=3 \times 10^{8}(\mathrm{~m} / \mathrm{s})$,

$G=6.672 \times 10^{-11}\left(N \cdot \mathrm{m}^{2} / \mathrm{kg}^{2}\right), B$ is a magnetic field in Tesla and $R^{00}$ is a major component of spatial curvature $\left(1 / m^{2}\right)$.

On the other hand, Levi-Civita also investigated the gravitational field produced by a homogeneous electric or magnetic field, which was expressed by Pauli [16]. If $x^{3}$ is taken in the direction of a magnetic field of intensity F (Gauss unit), the square of the line element is of the form:

$$
\begin{aligned}
& d s^{2}=\left(d x^{1}\right)^{2}+\left(d x^{2}\right)^{2}+\left(d x^{3}\right)^{2}+\frac{\left(x^{1} d x^{1}+x^{2} d x^{2}\right)^{2}}{a^{2}-r^{2}} \\
& -\left[c_{1} \exp \left(x^{3} / a\right)+c_{2} \exp \left(-x^{3} / a\right)\right]^{2}\left(d x^{4}\right)^{2}
\end{aligned}
$$

where $\mathrm{r}=\sqrt{\left(x^{1}\right)^{2}+\left(x^{2}\right)^{2}}, c_{1}$ and $c_{2}$ are constants, $a=\frac{c^{2}}{\sqrt{k} F}, k$ is Newtonian gravitational constant $(\mathrm{G})$, and $x^{1} \ldots x^{4}$ are Cartesian coordinates $\left(x^{1} \ldots x^{3}=\right.$ space, $\left.x^{4}=c t\right)$ with orthographic projection.

The space is cylindrically symmetric about the direction of the field, and on each plane perpendicular to the field direction the same geometry holds as in Euclidean space on a sphere of radius $a$, that is, the radius of curvature $a$ is given by

$$
a=\frac{c^{2}}{\sqrt{k} F}
$$

Since the relation of between magnetic field B in SI units and magnetic field F in CGS Gauss units are described as follows: $B \sqrt{\frac{4 \pi}{\mu_{0}}} \Leftrightarrow F$, then the radius of curvature " $a$ " in Eq. (A3) is expressed in SI units as the following (changing symbol, $k \rightarrow G, F \rightarrow B)$

$$
a=\frac{c^{2}}{\sqrt{G} F}=\frac{c^{2}}{\sqrt{G} \cdot B \sqrt{\frac{4 \pi}{\mu_{0}}}} \quad \approx\left(3.484 \times 10^{18} \frac{1}{B} \text { meters }\right)
$$

While, scalar curvature is represented by

$$
R^{00} \approx R=\frac{1}{a^{2}}=\frac{G B^{2} \frac{4 \pi}{\mu_{0}}}{c^{4}}=\frac{4 \pi G}{\mu_{0} c^{4}} B^{2}
$$

which coincides with (A1). 